\title{
Mott transition in a two-leg Bose-Hubbard ladder under an artificial magnetic field
}

\author{
Ahmet Keleşs, ${ }^{1,2}$ and M. Ö. Oktel ${ }^{3, \dagger}$ \\ ${ }^{1}$ Department of Physics and Astronomy, University of Pittsburgh, Pittsburgh, Pennsylvania 15260, USA \\ ${ }^{2}$ School of Physics and Astronomy and Computational Sciences, George Mason University, Fairfax, Virginia 15260, USA \\ ${ }^{3}$ Department of Physics, Bilkent University, 06800 Ankara, Turkey
}

(Received 3 November 2014; published 28 January 2015)

\begin{abstract}
We consider the Bose-Hubbard model on a two-leg ladder under an artificial magnetic field and investigate the superfluid-to-Mott insulator transition in this setting. Recently, this system has been experimentally realized [M. Atala et al., Nature Phys. 10, 588 (2014)], albeit in a parameter regime that is far from the Mott transition boundary. Depending on the strength of the magnetic field, the single-particle spectrum has either a single ground state or two degenerate ground states. The transition between these two phases is reflected in the many-particle properties. We first investigate these phases through the Bogoliubov approximation in the superfluid regime and calculate the transition boundary for weak interactions. For stronger interactions the system is expected to form a Mott insulator. We calculate the Mott transition boundary as a function of the magnetic field and interleg coupling with mean-field theory, strong-coupling expansion, and density matrix renormalization group (DMRG). Finally, using the DMRG, we investigate the particle-hole excitation gaps of this system at different filling factors and find peaks at simple fractions, indicating the possibility of correlated phases.
\end{abstract}

DOI: 10.1103/PhysRevA.91.013629

PACS number(s): 03.75.Lm, 05.30.Jp, 05.70.Fh, 67.25.dj

\section{INTRODUCTION}

Cold-atom experiments can realize the fundamental models of many-particle physics which are not accessible with traditional condensed matter techniques. One recent advance has been the demonstration of artificial magnetic fields in optical lattice systems, as well as in continuums [1-3]. The optical lattice experiments control the phase of the hopping between lattice sites to create a Hamiltonian with an artificial magnetic field. This effective magnetic feld is orders of magnitude larger than what is attainable in a solid-state experiment. For the typical lattice constants in solids, the magnetic flux through a unit cell is comparable to the flux quantum $h / e$ only for magnetic fields in excess of thousands of teslas. The first experiments demonstrating effective magnetic fields in optical lattices have proven that this extremely high magnetic field regime is accessible with cold atoms [2,3].

Most investigations of magnetic-field effects in many particle systems rely on a separation of length scales, assuming that the magnetic length is much larger than the lattice scale. However, if these two length scales are comparable, the magnetic field can no longer be treated semiclassically and has to be directly taken into account in the microscopic Hamiltonian. The profound effect of such strong magnetic fields can be observed even for noninteracting particles. The single-particle spectrum is sensitively dependent on the external field, forming a self-similar structure known as the Hofstadter butterfly [4]. Recent experiments hold the promise of investigation of many-particle physics for systems with such complicated single-particle dispersions. The interplay between interactions and the complicated single-particle spectrum is expected to result in novel phases $[5,6]$.

The first experiments which implemented an artificial magnetic field for lattice systems demonstrated the existence

\footnotetext{
*ahmetkeles99@gmail.com

†oktel@fen.bilkent.edu.tr
}

of the artificial magnetic field by measuring the effect of this field on excited states of the system [2,3]. Thus they did not probe the ground state of the Hofstadter-Hubbard Hamiltonian. The recent experiment by the Munich group has, for the first time, demonstrated the effects of an artificial magnetic field on the ground state of a lattice system.

The experiment in Ref. [7] realizes a model which is essentially one-dimensional. In general, the orbital coupling of the magnetic field to a one-dimensional system does not create any change, as such a field can be set to 0 by a gauge transformation. However, by using a two-leg ladder, the experiment creates a situation in which the magnetic field has nontrivial effects on the system without generating a complicated single-particle spectrum or a sensitive dependence on the rationality of the applied field. Thus, experimental realization of this system provides the first opportunity to study the behavior of lattice bosons in an extremely high magnetic field regime.

In this paper, we investigate this model system theoretically, particularly focusing on the effect of the artificial magnetic field on the Mott insulator-to-superfluid transition. We have previously conducted a theoretical study of the two-leg BoseHubbard ladder [8]. In this paper our unpublished results are summarized and extended to cover the regime investigated by the experiment.

We find that the transition between the Meissner and the vortex phases moves to a higher magnetic field for weak interactions. For strong interactions the system goes into the Mott insulator state. We find consistent results from the strongcoupling expansion and density matrix renormalization group (DMRG) for the Mott insulator boundary. A magnetic field stabilizes the Mott state and makes it accessible at a lower interaction strength. We also find that there is a re-entrant Mott transition as a function of the hopping strength at fixed chemical potential. Finally, we investigate the gap between the ground and the first excited states through the DMRG and find that there are distinct peaks at simple filling fractions, providing evidence for the existence of correlated states in this system. 
The paper is organized as follows: We introduce the Hamiltonian in Sec. II and review the properties of the single-particle spectrum in Sec. III. In Sec. IV, we investigate the system with weak interactions using the Gross-Pitaevskii mean-field approximation and also discuss the excitations of the system above the mean-field solution. The remaining sections focus on the strongly interacting regime. In Sec. V, we calculate the phase diagram of the system using a real-space Gutzwiller ansatz. This approximation is particularly poor for one-dimensional systems, thus in Sec. VI we calculate the phase diagram using strong-coupling perturbation theory. Section VII contains a discussion of the Mott transition using the DMRG. In Sec. VIII, we investigate the gap between the ground and the first excited state of the system at half-filling in the infinite interaction limit as well as the gaps in the particle-hole excitations of the system for various fillings using the DMRG and discuss the possibility of correlated states. Finally, we summarize our results and their consequences for experiments in Sec. IX.

\section{MODEL}

We consider an infinite ladder composed of square plaquettes extending in the $\hat{x}$ direction, with nearest-neighbor hopping. The tight-binding Hamiltonian for this two-leg ladder is given by

$$
\begin{aligned}
H= & -\sum_{i}\left[J e^{-i \alpha} a_{i}^{\dagger} a_{i+1}+J e^{i \alpha} b_{i}^{\dagger} b_{i+1}+K a_{i}^{\dagger} b_{i}+\text { H.c. }\right] \\
& +\frac{U}{2} \sum_{i} n_{i}^{a}\left(n_{i}^{a}-1\right)+n_{i}^{b}\left(n_{i}^{b}-1\right)-\mu \sum_{i} n_{i}^{a}+n_{i}^{b},
\end{aligned}
$$

where $a_{i}, b_{i}\left(a_{i}^{\dagger}, b_{i}^{\dagger}\right)$ are bosonic annihilation (creation) operators for the $i$ th site in the upper and lower legs, respectively. $n_{i}^{a}=a_{i}^{\dagger} a_{i}$ and $n_{i}^{b}=b_{i}^{\dagger} b_{i}$ are the corresponding number operators, $J(K)$ is the intraleg (interleg) hopping strength, $U$ is the on-site interaction strength, and $\mu$ is the chemical potential. We assume a homogeneous system that has "up-down" symmetry for zero magnetic field, so that on-site interactions and chemical potentials are identical for each leg. The phase $\alpha$ accumulated by hopping from $\mathbf{r}_{i}$ to $\mathbf{r}_{j}$ is

$$
\alpha=\frac{e}{\hbar} \int_{\mathbf{r}_{\mathbf{i}}}^{\mathbf{r}_{\mathbf{j}}} d \mathbf{r} \cdot \mathbf{A}(\mathbf{r}),
$$

where $\mathbf{A}$ is the vector potential satisfying $\nabla \times \mathbf{A}=\mathbf{B}$ and $\mathbf{B}$ is the magnetic field perpendicular to the two-leg plane. We use the Landau gauge $\mathbf{A}=-B y \hat{x}$ for $\mathbf{B}=B \hat{z}$ and choose $y=0$ to be at the center of two legs so that the upper and lower legs will be at positions $y=c / 2$ and $y=-c / 2$, respectively. Thus, the exponent in Eq. (1) can be calculated from Eq. (2) as $\alpha=\pi \phi / \phi_{0}$, where $\phi$ is the magnetic flux passing through each plaquette and $\phi_{0}=h / e$ is the flux quantum.

The main advantage of considering a two-leg ladder as opposed to a two-dimensional extended system is immediately obvious. For two-dimensional systems, periodicity under translations can only be obtained when $\phi / \phi_{0}$ is taken to be a rational number $p / q$. Only then can the symmetry broken by the specific gauge choice be restored in a $q$-fold enlarged
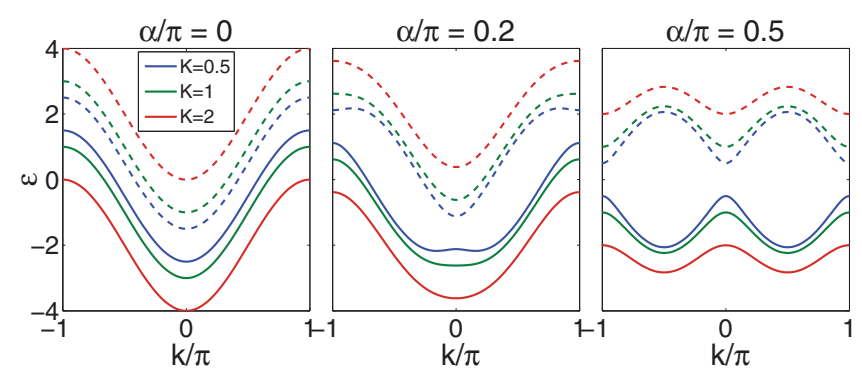

FIG. 1. (Color online) Single-particle spectrum of a two-leg ladder with varying magnetic flux $\alpha$ and interleg-to-intraleg hopping ratio $K$. Lower bands are shown by solid lines, whereas upper bands are shown by dashed lines. The bottom (red) solid line is for $K=2$, the middle (green) solid line is for $K=1$, and the top (blue) solid line is for $K=0.5$. The gap between lower and higher bands that appears for $\alpha / \pi=0.5$ is further shown for the case $K=1$ as a function of the magnetic field in Fig. 2. It is also observed that the gap around $k=0$ for $\alpha / \pi=0.5$ closes for very small $K \ll 1$, giving rise to a linear dispersion around $k=0$.

unit cell. The two-leg ladder system does not require such a constraint so that calculations can be carried out for any real number $\alpha / \pi$ between 0 and 1 . As such, the two-leg system presents an opportunity to observe the nontrivial effects of an external field in a lattice system without the added theoretical complication. The profound effect of the magnetic field is evident even at the single-particle level, which is presented in the next section.

\section{SINGLE-PARTICLE SOLUTION}

We first give solutions for noninteracting particles; $U=0$. Using the translational invariance along the $x$ direction, the Fourier components of the field operators can be written as

$$
a_{j}=\frac{1}{\sqrt{L}} \sum_{k} a_{k} e^{i k j}, \quad b_{j}=\frac{1}{\sqrt{L}} \sum_{k} b_{k} e^{i k j},
$$

where the Fourier components satisfy the commutation $\left[a_{k}, a_{k^{\prime}}^{\dagger}\right]=\delta_{k k^{\prime}}$ and $\left[b_{k}, b_{k^{\prime}}^{\dagger}\right]=\delta_{k k^{\prime}}$, all other commutators being 0 . For simplicity, we have taken $c=1$ above so that all lengths are measured in units of the lattice constant. Using these transformations in Eq. (1), the following Hamiltonian can be obtained in the momentum space

$$
H_{\mathrm{sp}}=-\sum_{k}\left[\xi_{a k} a_{k}^{\dagger} a_{k}+\xi_{b k} b_{k}^{\dagger} b_{k}+K a_{k}^{\dagger} b_{k}+K b_{k}^{\dagger} a_{k}\right],
$$

where $\xi_{a k}$ and $\xi_{b k}$ are $2 J \cos (k-\alpha)$ and $2 J \cos (k+\alpha)$, respectively. Diagonalization is achieved by the Bogoliubov transformation $\quad A_{k}=\cos \theta a_{k}+\sin \theta b_{k}, \quad B_{k}=-\sin \theta a_{k}+$ $\cos \theta b_{k}$, where $\theta=\frac{1}{2} \arctan \left(\frac{2 K}{\xi_{a k}-\xi_{b k}}\right)$. The energy eigenvalues $\epsilon_{1,2}$ can be found as

$$
\epsilon_{1,2}=-2 \cos (k) \cos (\alpha) \mp \sqrt{\tilde{K}^{2}+4 \sin ^{2} k \sin ^{2} \alpha},
$$

where $\tilde{K}=K / J$ and we normalize the energy with the interleg hopping $J$. In Fig. 1, we show the dispersion relation in the first Brillouin zone, for zero and nonzero magnetic fields. It can be seen that, as the strength of the field increases, the band minimum in the dispersion shifts from $k=0$ to two nonzero 

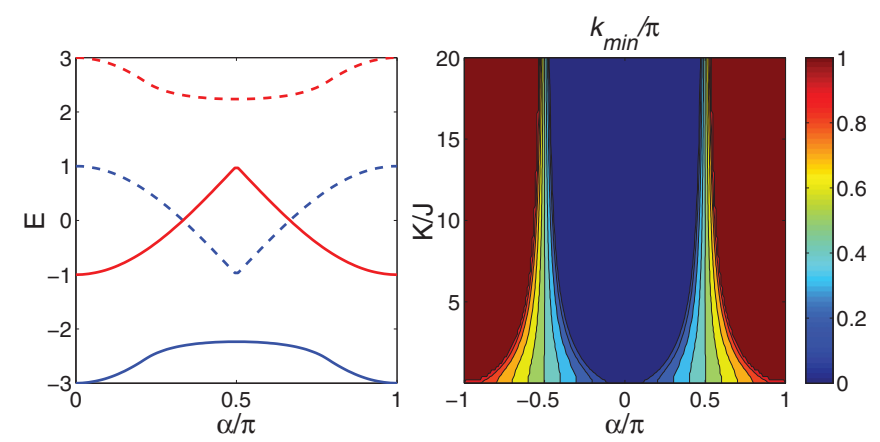

FIG. 2. (Color online) Left: Minima and maxima for the two bands as a function of the magnetic field. Lower solid and lower dashed (blue) lines are the minimum and maximum of the lower band, respectively. Similarly, upper solid and upper dashed (red) lines are the minimum and maximum of the the higher band. The band gap is evident between $\alpha / \pi=1 / 3$ and $\alpha / \pi=2 / 3$, which attains its maximum value at $\alpha / \pi=1 / 2$. Right: Value of the reciprocal lattice vector at the minimum energy as a function of the magnetic field and the hopping parameter $K / J$.

$k$ values that are degenerate and symmetric around the origin. The critical field for this bifurcation depends on the parameter $\tilde{K}$ as

$$
\alpha_{c}=\cos ^{-1}\left(-\frac{\tilde{K}}{4} \pm \sqrt{\frac{\tilde{K}^{2}}{16}+1}\right)
$$

Above this critical field, the ground state of the system will no longer be spatially uniform but will be a superposition of the plane waves corresponding to the two minima, which can be found from the dispersion as

$$
k_{\min }= \pm \sin ^{-1} \sqrt{\sin ^{2} \alpha-\frac{\tilde{K}^{2}}{4 \tan ^{2} \alpha}} .
$$

In the Munich experiment [7], these two ground states were observed for weakly interacting bosons and have been named the Meissner and vortex phases, respectively.

As shown in Fig. 1, for small values of the magnetic field, there is no gap between the lower band and the upper band, whereas for $\alpha / \pi=0.5$ there is a finite band gap between these two and it decreases as $K / J$ is reduced. We observe that this gap closes as $K / J \rightarrow 0$ and a singular point emerges at $k=0$ in this limit. To show more detailed behavior of the band gap, we plot the minimum and the maximum of the two bands as a function of the magnetic field for $K=J$ in Fig. 2. This plot can be regarded as the "Hofstadter butterfly" of the two-leg ladder system. We see that a diamond-shaped gapped region starts at $\alpha / \pi=1 / 3$, takes its maximum value $2 J$ at $\alpha / \pi=1 / 2$, and ends at $\alpha / \pi=2 / 3$. In Fig. 2, we also provide the value of the reciprocal lattice vector $k_{\min }$ as given in Eq. (7) at the band minimum as a function of the magnetic field and the parameter $K / J$, which is in agreement with [7].

\section{GROSS-PITAEVSKII APPROXIMATION}

Our picture of the transition between the Meissner and the vortex phases in the previous section depended only on the noninteracting single-particle spectrum of the two-leg ladder. Before we discuss the effects of strong interactions and the resulting insulating phase we concentrate on the weakly interacting limit and calculate how the Meissner-to-vortex transition is affected by the presence of interactions.

For small values of the interaction strength and the magnetic field, the system will essentially be in the superfluid state, mostly dominated by the hopping term in the Hamiltonian. Thus, assuming that the condensate fluctuations are negligible, we make the following approximation:

$$
a_{i} \rightarrow\left\langle a_{i}\right\rangle=\psi_{i}, \quad b_{i} \rightarrow\left\langle b_{i}\right\rangle=\phi_{i} .
$$

Both the amplitude and the phase of those classical fields are time and position dependent. Clearly, approximation with a uniform condensate will fail above the critical field.

Making substitution (8) in Eq. (1), the following energy functional is obtained (here we take $J=1$ so that $U, \mu$, and $K$ are in units of $J$ ):

$$
\begin{aligned}
E= & -\sum_{j}\left[e^{-i \alpha} \psi_{j}^{*} \psi_{j+1}+e^{i \alpha} \phi_{j}^{*} \phi_{j+1}+K \psi_{j}^{*} \phi_{j}+\text { c.c. }\right] \\
& +\frac{U}{2} \sum_{j}\left[\psi_{j}^{*} \psi_{j}\left(\psi_{j}^{*} \psi_{j}-1\right)+\phi_{j}^{*} \phi_{j}\left(\phi_{j}^{*} \phi_{j}-1\right)\right] \\
& -\mu \sum_{j}\left|\psi_{i}\right|^{2}+\left|\phi_{i}\right|^{2} .
\end{aligned}
$$

Variation of the energy functional around the minimal solutions $i \partial \psi_{i} / \partial t=\delta E / \delta \psi_{i}{ }^{*}$ and $i \partial \phi_{i} / \partial t=\delta E / \delta \phi_{i}^{*}$ gives the following coupled Gross-Pitaevskii equations:

$$
\begin{aligned}
i \frac{\partial \psi_{j}}{\partial t}= & -\left[e^{-i \alpha} \psi_{j+1}+K \phi_{j}+e^{i \alpha} \psi_{j-1}\right] \\
& +U\left|\psi_{j}\right|^{2} \psi_{j}-\left(\frac{U}{2}+\mu\right) \psi_{j} \\
i \frac{\partial \phi_{j}}{\partial t}= & -\left[e^{i \alpha} \phi_{j+1}+K \psi_{j}+e^{-i \alpha} \phi_{j-1}\right] \\
& +U\left|\phi_{j}\right|^{2} \phi_{j}-\left(\frac{U}{2}+\mu\right) \phi_{j} .
\end{aligned}
$$

Zeroth-order terms $\psi_{j}=\phi_{j}=\sqrt{n}$ give the chemical potential as $\mu=-(2 \cos \alpha+\tilde{K})+0.5 U(2 n-1)$. For a higher order approximation, the fluctuations in the condensate are taken into account as [9]:

$$
\begin{aligned}
& \psi_{j}=\sqrt{n}+A e^{i\left(k x_{j}-\omega t\right)}+B^{*} e^{-i\left(k x_{j}-\omega t\right)}, \\
& \phi_{j}=\sqrt{n}+C e^{i\left(k x_{j}-\omega t\right)}+D^{*} e^{-i\left(k x_{j}-\omega t\right)},
\end{aligned}
$$

where $A, B, C$, and $D$ are small complex parameters, $x_{j}$ is the position of the lattice site, and $k$ is the reciprocal lattice vector. Inserting these wave functions into Eq. (11), the equation of motion can be reduced to an algebraic equation of the form $H_{\mathrm{gp}} \vec{\Psi}=\omega \vec{\Psi}$ where $\vec{\Psi}=(A, B, C, D)$ and $H_{\mathrm{gp}}$ has the form

$$
H_{\mathrm{gp}}=\left[\begin{array}{cccc}
-\xi_{a k}^{\prime} & U n & -K & 0 \\
-U n & \xi_{b k}^{\prime} & 0 & K \\
-K & 0 & -\xi_{b k}^{\prime} & U n \\
0 & K & -U n & \xi_{a k}^{\prime}
\end{array}\right],
$$



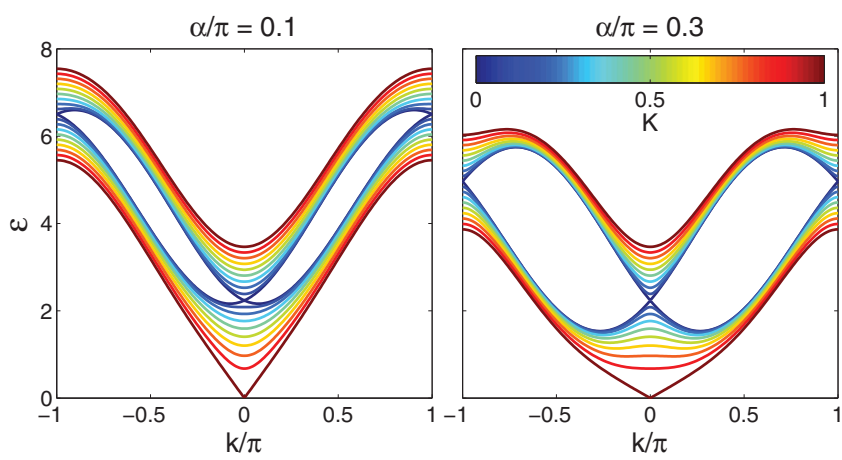

FIG. 3. (Color online) Band diagrams for a two-leg ladder with on-site interactions calculated within the Gross-Pitaevskii approximation for $U=2$. Left: $\alpha / \pi=0.1$. Right: $\alpha / \pi=0.3$.

where $\xi_{a k}^{\prime}=2 \cos (k-\alpha)-2 \cos (\alpha)-U n-K$ and $\xi_{b k}^{\prime}=$ $2 \cos (k+\alpha)-2 \cos (\alpha)-U n-K$. The resulting change in the spectrum can be obtained by calculating the eigenvalue of $H_{\mathrm{gp}}$, which is shown in Fig. 3.

Competition between the magnetic field and the interactions can be seen by considering the band minima around $k=0$ in Fig. (3). Here the interactions sharpen the band and provide a cusp-like shape, whereas the increase in the magnetic field makes it smoother.

The expansion of the wave function in Eq. (12) fails above the critical magnetic field, as the ground state is no longer spatially uniform, and the eigenvalues of $H_{g p}$ start having imaginary parts. We have used this property to determine the change in the critical field with the interaction strength. In Fig. 4, the critical magnetic field as a function of the strength of the interaction is shown. It can be seen that the $U-\alpha_{c}$ relation is almost linear for low interaction strengths but saturates for strong interactions. It must be noted that for strong interactions Gross-Pitaevskii approximation is not reliable.

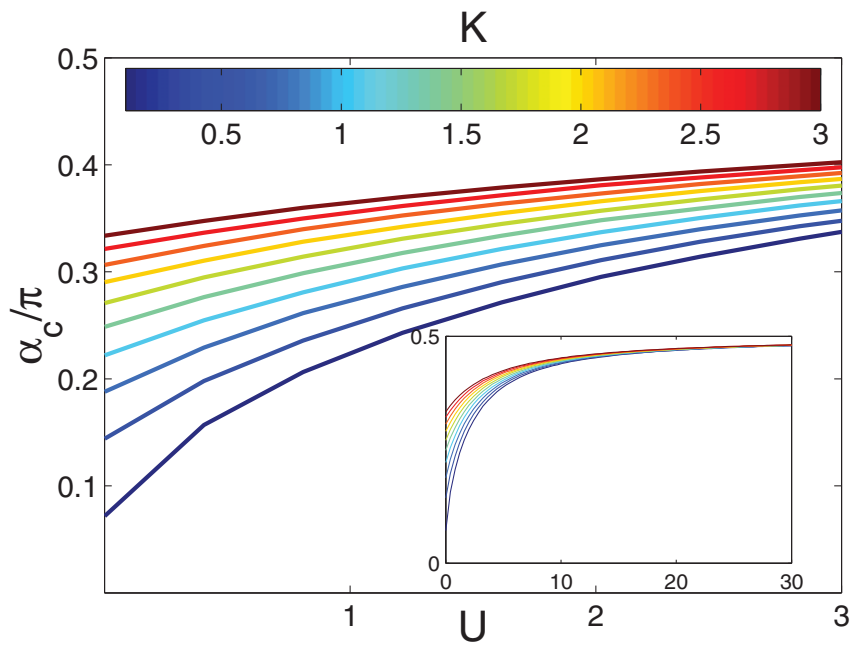

FIG. 4. (Color online) Critical magnetic field plotted as a function of the interaction strength $U$ and the intraleg hopping $K$. Inset: The same plot zoomed out to span large interactions. Note that the Gross-Pitaevskii approximation is not expected to be reliable for strong interactions.

\section{VARIATIONAL MEAN-FIELD APPROACH}

In this section, we consider the transition from the superfluid state to the Mott insulating state as a function of $J, K$, $\mu$, and $\alpha$. Here, it is convenient to scale the Hamiltonian in Eq. (1) with $U=1$. In the perfect Mott insulator phase, each site has a localized wave function with exactly $n_{0}$ particles such that the wave function in each site is $\left|n_{0}\right\rangle_{i}$ in the Fock basis. Allowing small variations around this equilibrium, we write the Gutzwiller ansatz for local sites,

$$
\begin{aligned}
|G\rangle_{a k} & =\Delta_{a k}\left|n_{0}-1\right\rangle_{a k}+\left|n_{0}\right\rangle_{a k}+\Delta_{a k}^{\prime}\left|n_{0}+1\right\rangle_{a k}, \\
|G\rangle_{b k} & =\Delta_{b k}\left|n_{0}-1\right\rangle_{b k}+\left|n_{0}\right\rangle_{b k}+\Delta_{b k}^{\prime}\left|n_{0}+1\right\rangle_{b k},
\end{aligned}
$$

where $\Delta$ and $\Delta^{\prime}$ are small complex variational parameters. The wave function for a rung is $|G\rangle_{r k}=|G\rangle_{a k}|G\rangle_{b k}$ so that the total wave function of the system can be written as $|\Psi\rangle=\prod_{k}|G\rangle_{a k}|G\rangle_{b k}$. The variational energy of the system is calculated from $\varepsilon=\langle\Psi|H| \Psi\rangle /\langle\Psi \mid \Psi\rangle$ up to second order in $\Delta$ and $\Delta^{\prime}$ as follows:

$$
\begin{aligned}
\varepsilon= & \sum_{i=1}^{N}\left\{-J e^{-i \alpha}\left[n_{0} \Delta_{a, i} \Delta_{a, i+1}^{*}+\left(n_{0}+1\right) \Delta_{a, i}^{\prime *} \Delta_{a, i+1}^{\prime}\right.\right. \\
& \left.+\sqrt{n_{0}\left(n_{0}+1\right)} \Delta_{a, i} \Delta_{a, i+1}^{\prime}+\sqrt{n_{0}\left(n_{0}+1\right)} \Delta_{a, i}^{\prime *} \Delta_{a, i+1}^{*}\right] \\
& -J e^{i \alpha}[a \rightarrow b]-K\left[n_{0} \Delta_{a, i} \Delta_{b, i}^{*}+\left(n_{0}+1\right) \Delta_{a, i}^{* *} \Delta_{b, i}^{\prime}\right. \\
& \left.+\sqrt{n_{0}\left(n_{0}+1\right)}\left(\Delta_{a, i} \Delta_{b, i}^{\prime}+\Delta_{a, i}^{*} \Delta_{b, i}^{*}\right)\right] \\
& +\left[\left(1-n_{0}+\mu\right)\left(\left|\Delta_{a, i}\right|^{2}+\left|\Delta_{b, i}\right|^{2}\right)+n_{0}\left(n_{0}-1-2 \mu\right)\right. \\
& \left.\left.+\left(n_{0}-\mu\right)\left(\left|\Delta_{a, i}^{\prime}\right|^{2}+\left|\Delta_{b, i}^{\prime}\right|^{2}\right)\right]\right\} .
\end{aligned}
$$

We minimize the energy with respect to $\Delta_{a i}, \Delta_{b i}, \Delta_{a i}^{\prime}$, and $\Delta_{b i}^{\prime}$. The Jacobian matrix of the second derivatives is calculated as

$$
\begin{aligned}
\mathbf{J}= & -\left(\begin{array}{cc}
n_{0} \mathbf{F} & \sqrt{n_{0}\left(n_{0}+1\right)} \mathbf{F} \\
\sqrt{n_{0}\left(n_{0}+1\right)} \mathbf{F} & \left(n_{0}+1\right) \mathbf{F}
\end{array}\right) \\
& +\left(\begin{array}{cc}
\left(1-n_{0}+\mu\right) \mathbf{I} & 0 \\
0 & \left(n_{0}-\mu\right) \mathbf{I}
\end{array}\right),
\end{aligned}
$$

where $\mathbf{I}$ is a $2 N \times 2 N$ identity matrix and $\mathbf{F}$ is written as

$$
\mathbf{F}=\left[\begin{array}{cccc}
A & B & \ldots & B^{\dagger} \\
B^{\dagger} & A & \ddots & 0 \\
\vdots & \ddots & \ddots & B \\
B & 0 & B^{\dagger} & A
\end{array}\right]
$$

Here sub-blocks are defined in terms of Pauli matrices in the upper leg-lower leg basis as

$$
A=K \sigma_{x}, \quad B=J e^{i \alpha \sigma_{z}} .
$$

To find the eigenvalues, we use the same method presented in Ref. [6]: let $\lambda_{\mathbf{F}}$ and $\vec{u}$ be the eigenvalues and the eigenvectors of $\mathbf{F}$, respectively; then one can apply an ansatz of the form $\vec{v}=$ $(a \vec{u}, b \vec{u})$ and solve the eigenvalue equation $\mathbf{J} \vec{v}=\lambda \vec{v}$, which is 
found to be

$$
\lambda_{1,2}=1-\lambda_{\mathbf{F}}\left(2 n_{0}+1\right) \pm \sqrt{\left(1-\lambda_{\mathbf{F}}\left(2 n_{0}+1\right)\right)^{2}+4 \lambda_{\mathbf{F}}(\mu+1)-4\left(n_{0}-\mu\right)\left(1-n_{0}+\mu\right)} .
$$

Equating the minimum eigenvalue of the Jacobian matrix in Eq. (19) to 0 yields the phase boundary of the Mott insulating region. Solving the corresponding equation for $K$ and $J$, the following simple relation can be found for the boundary of the Mott phase:

$$
\lambda_{\mathbf{F}}\left(K_{c}, J_{c}\right)=\frac{\left(n_{0}-\mu\right)\left(1-n_{0}+\mu\right)}{(\mu+1)} .
$$

Here $\lambda_{\mathbf{F}}$ is the minimum value of $\epsilon_{1}$ in Eq. (5) so that we obtain the Mott phase boundary for each value of the magnetic field $\alpha$. In Fig. 5, Eq. (20) is plotted for $n_{0}=1$, which shows the shape of the Mott insulation region.

Note that this result is exact within the mean-field theory. However, the mean-field theory in a quasi-one-dimensional system is not expected to be accurate. The decoupling of the hopping term in Eq. (1) upon introducing a mean field is questionable in a low-dimensional system, where the effect of fluctuations is necessarily important. The mean-field calculation can only describe the system at a qualitative level. It provides a general idea about the topology of the Mott region and an estimate of the phase boundary for small values of the hopping strength where site-site correlations are diminished. For a better determination of the Mott insulating region, we turn to more accurate methods in the following sections.

\section{STRONG-COUPLING EXPANSION}

A better description of the transition is obtained by treating the hopping term as a perturbation in the perfect Mott state. While this is, in spirit, close to the mean-field approach given in the previous section, correlations between the sites are built in as higher orders in perturbation theory are developed. The resulting "strong-coupling expansion" has been successfully applied to the Bose-Hubbard model in low dimensions and has been shown to be in perfect agreement with accurate numerical methods [10,11].

In the strong-coupling expansion, the hopping amplitude is considered as a small parameter. The Mott insulator state is characterized by a finite gap for particle-hole excitations, whereas this gap vanishes for the superfluid phase [12]. We calculate the energy of a system with exactly $n_{0}$ particles per site (Mott state $E_{M}$ ) and the energy of a system with one additional defect (particle $E_{P}$ or hole $E_{H}$ ) perturbatively. The energy difference between the defect states and the perfect Mott state vanishes at the phase boundary. This method has been used for systems with different dimensions $[13,14]$ and for a two-dimensional system under a magnetic field [15].

For calculations under perturbation theory, it is convenient to write the Hamiltonian in the generalized form,

$$
H=-\sum_{i j} \mathbf{F}_{i j} \tilde{a}_{i}^{\dagger} \tilde{a}_{j}+\frac{1}{2} \sum_{i} \tilde{n}_{i}\left(\tilde{n}_{i}-1\right)-\mu \sum_{i} \tilde{n}_{i},
$$

where $\mathbf{F}$ is given in Eq. (17) for our model and the correspondence between the operators $\tilde{a}_{i}$ and $\tilde{n}_{i}$ and the operators in Eq. (1) is obvious.

We perform strong-coupling perturbation up to second order in our calculations. The energies of the Mott state $E_{M}$, the additional particle state $E_{P}$, and the additional hole state $E_{H}$ are found to be

$$
\begin{aligned}
E_{M}= & E_{M}^{0}-N n_{0}\left(n_{0}+1\right)\left(2 J^{2}+K^{2}\right) \\
E_{P}= & E_{P}^{0}-\left(n_{0}+1\right) \lambda_{\mathbf{F}}-N n_{0}\left(n_{0}+1\right)\left(2 J^{2}+K^{2}\right) \\
& -n_{0}\left(n_{0}+1\right) \lambda_{\mathbf{F}}^{2}+\frac{1}{2} n_{0}\left(5 n_{0}+4\right)\left(2 J^{2}+K^{2}\right) \\
E_{H}= & E_{H}^{0}-n_{0} \lambda_{\mathbf{F}}-N n_{0}\left(n_{0}+1\right)\left(2 J^{2}+K^{2}\right) \\
& -n_{0}\left(n_{0}+1\right) \lambda_{\mathbf{F}}^{2}+\frac{1}{2}\left(n_{0}+1\right)\left(5 n_{0}+1\right)\left(2 J^{2}+K^{2}\right),
\end{aligned}
$$

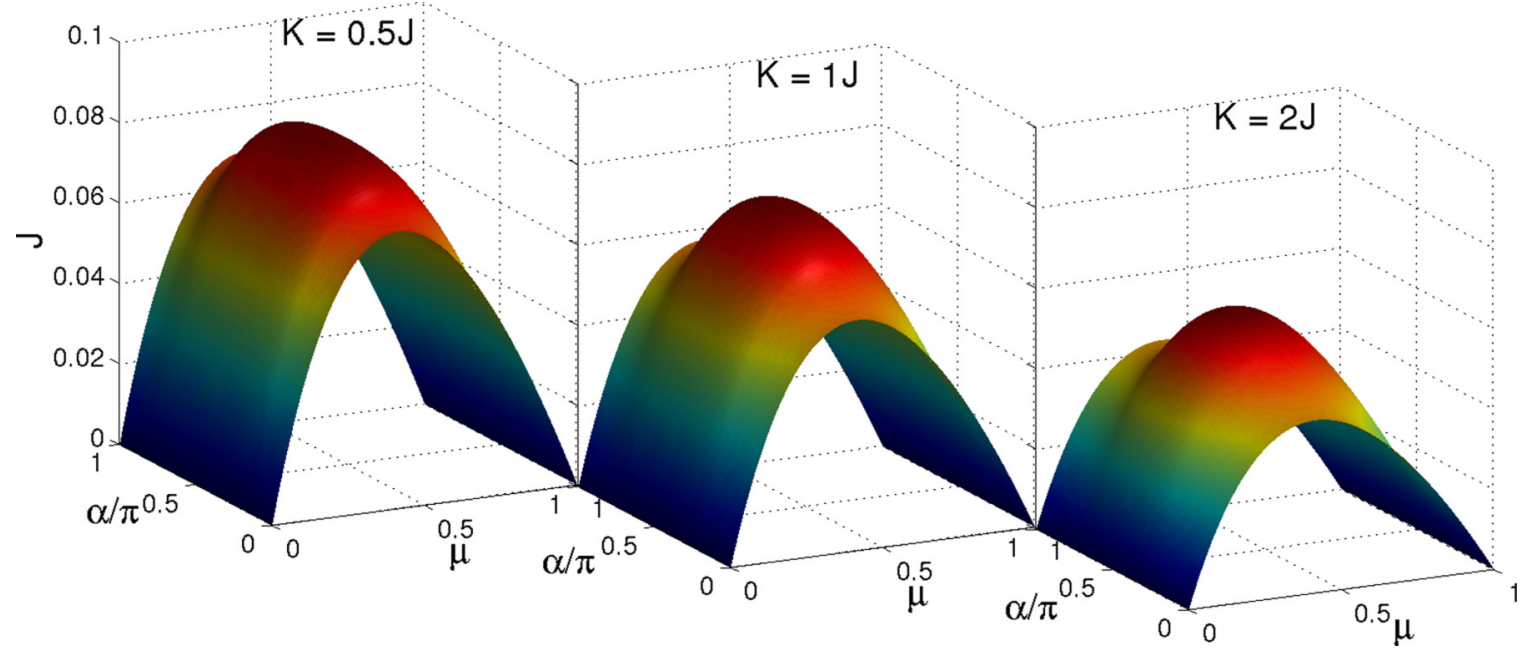

FIG. 5. (Color online) Mott insulating phase boundary calculated within the variational mean-field approach as a function of the magneticfield strength and the chemical potential for the parameters $K=0.5 J, K=J, K=2 J$. The region below (above) the plotted surface is the insulating (superfluid) state. 

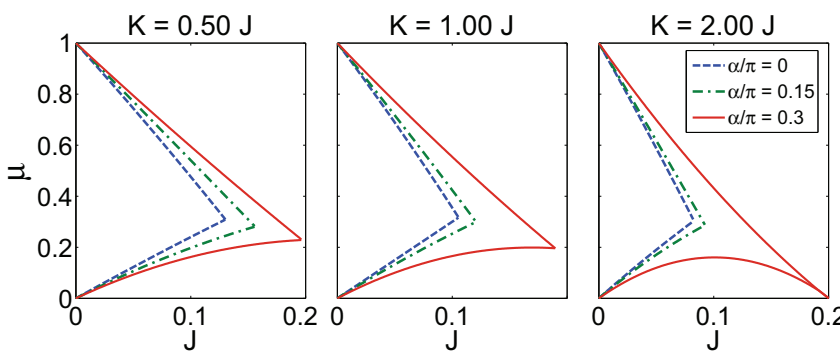

FIG. 6. (Color online) Phase diagram of a two-leg ladder from the strong-coupling expansion up to second order for different magnetic fields and interleg-to-intraleg hopping ratios.

where $\lambda_{\mathbf{F}}$ is the lowest eigenvalue of hopping matrix $\mathbf{F}$ and $N$ is the number of lattice sites in one leg. Zeroth-order energies are $E_{M}^{0}=2 N\left(n_{0}\left(n_{0}-1\right) / 2-\mu n_{0}\right), E_{P}^{0}=E_{M}^{0}+$ $n_{0}-\mu$, and $E_{H}^{0}=E_{M}^{0}-\left(n_{0}-1\right)+\mu$. Solving the equations $E_{P}-E_{M}=0$ and $E_{M}-E_{H}=0$ for the chemical potential $\mu$ separately, the phase boundary of the particle sector and hole sector is obtained as

$$
\begin{aligned}
\mu_{P}= & n_{0}+\left(n_{0}+1\right) \lambda_{\mathbf{F}}-n_{0}\left(n_{0}+1\right) \lambda_{\mathbf{F}}^{2} \\
& +\frac{1}{2} n_{0}\left(5 n_{0}+4\right)\left(2 J^{2}+K^{2}\right), \\
\mu_{H}= & \left(n_{0}-1\right)-n_{0} \lambda_{\mathbf{F}}-n_{0}\left(n_{0}+1\right) \lambda_{\mathbf{F}}^{2} \\
& -\frac{1}{2}\left(n_{0}+1\right)\left(5 n_{0}+1\right)\left(2 J^{2}+K^{2}\right) .
\end{aligned}
$$

Here the magnetic-field dependence comes indirectly from the eigenvalue $\lambda_{\mathbf{F}}$, but higher order terms in the perturbation will depend on the magnetic field explicitly. An interesting observation is that our results to this order are similar to the results in Ref. [15] for a number of nearest neighbors equal to 3. However, this is not guaranteed for higher order expansions since the flux attained through hopping is different due to the difference in the topology of this constrained problem. The eigenvalue spectrum of the $\mathbf{F}$ matrix is shown in Fig. 2.

In Fig. 6 we show the results of this calculation. An increase in the magnetic field enlarges the Mott insulating region of the phase diagram. This is expected as the magnetic field localizes the single-particle trajectories even for the noninteracting problem, thus a transition to an insulator state is easier. The Mott lobe grows in size until $\alpha=0.5$ and then reduces to satisfy periodicity at $\alpha=1$. The shape of the lobe is not concave as predicted by the mean field, but convex with a cusp at the tip. This shape is generic in one dimension, as obtained by strong-coupling, Monte Carlo, and DMRG results in one dimension. Comparing Figs. 5 and 6, it can be observed that the mean-field results underestimate the Mott boundary by a considerable amount.

A new feature of the phase diagram emerges after $\alpha=0.3$. The Mott phase has a re-entrance as a function of the hopping strength at fixed chemical potential. (Beyond $\alpha=0.3$ for $K=2$, curves of the particle and the hole sector intersect at such a large value of the hopping amplitude that the second-order perturbation theory fails to capture this region.) The behavior of the phase diagram here can be explained as follows. At a fixed chemical potential, if we start from the pure Mott state, one expects the excitations above this state to be mobile, as they move on a constant background of filled sites. If the kinetic energy gained by this mobility compensates the interaction energy with the background, these excitations become energetically favorable and cause the Mott insulator-to-superfluid transition. The magnetic field modifies this usual transition mainly by limiting the mobility of the excitations; this is why, in higher dimensional Bose-Hubbard models, the magnetic field increases the size of the Mott lobes in the phase diagram. In the two-leg ladder this confining effect takes an interesting form: starting from the pure Mott state, introduction of a small hopping causes the system to become superfluid, however, further increase in the hopping strength localizes the excitations and causes the system to go back to the Mott insulating state. Thus, for small $J$ the excitation energies are not affected by the magnetic field, but as hopping is increased this term becomes dominant and causes a phase transition back to the insulator phase. As J is increased further the system is once again dominated by kinetic energy and reaches the superfluid state.

The re-entrant phase behavior found in one-dimensional systems appears in the two-leg ladder with an increase in the magnetic field. This re-entrant behavior was not observed in the results for strong-coupling perturbation in one, two, or three dimensions or in a two-dimensional lattice under a magnetic field (in [13]-[15] perturbation was carried out up to third order). The existence of this re-entrant phase is also supported by our DMRG results, which is the subject of the next section.

\section{DMRG CALCULATIONS}

DMRG theory have been proven to provide numerically exact solutions of one-dimensional lattice systems $[16,17]$. This method has been extensively applied to the Bose-Hubbard model $[10,11,18]$ and shown to be one of the most reliable approaches for quasi-one-dimensional systems. Thus, in this section, we use the DMRG to calculate the Mott transition boundary for a two-leg Bose-Hubbard ladder under a magnetic field.

We use a method similar to that in [19], namely, rung-byrung enlargement, but employ single-rung enlargement [20] in the construction of the superblock Hamiltonian. We use the finite-system DMRG algorithm for a ladder of 60 rungs and for each site we set the maximum occupancy $n_{\max }=4$. Particle number conservation is used to diagonalize only the $N_{\text {particle }}=N_{\text {sites }}$ sector of the superblock Hamiltonian or $N_{\text {particle }}=N_{\text {sites }} \pm 1$ as additional target states. Further details about the projection to the space with different fillings are given in the next section.

Calculation of the Mott phase boundary via DMRG is very similar to the strong-coupling perturbation method. One needs the energies of the Mott phase together with the additional particle and hole states to find the phase boundary. The energies of particle and hole states are calculated as additional target states in the DMRG implementation [10]. In Fig. 7, one can see the good agreement between the strong-coupling result and the DMRG. For larger values of hopping, the strongcoupling method deviates from the DMRG, as expected for a perturbative method. Another point is that the existence of reentrance is also validated by DMRG results. We show a similar 

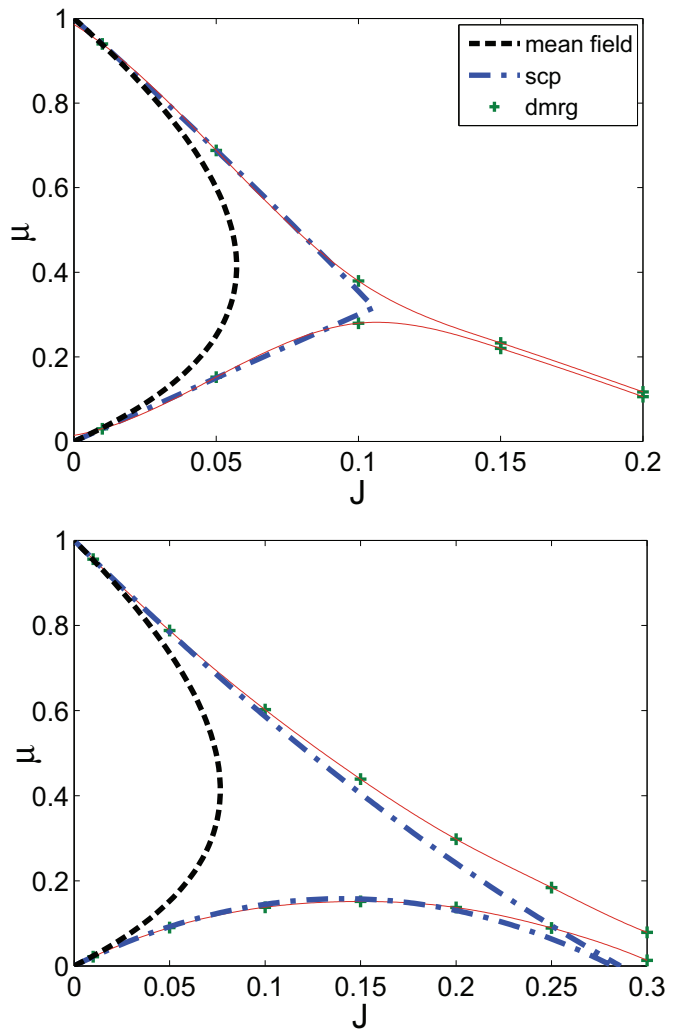

FIG. 7. (Color online) Phase diagram of a two-leg Bose-Hubbard ladder from the mean-field, strong-coupling expansion and DMRG at $\alpha=0$ (top) and at $\alpha / \pi=0.45$ (bottom). The thin solid (red) line is the spline interpolation to DMRG data points.

phase for $\alpha=0.45$ in Fig. 7. It is seen that strong-coupling calculations give relatively poor results above $J \approx 0.2$.

Finally, we note that the tip of the Mott insulator region requires a special treatment with the DMRG. The two branches coming from the particle and hole sector intersect only in the thermodynamic limit, whereas our system is composed of only 60 rungs. There are several approaches (like consideration of the correlation length and extrapolation to the Luttinger liquid correlation function in [10]) to remedying this situation. As the critical behavior of the tip is not our main concern in this paper, we do not perform a similar analysis.

\section{EVIDENCE OF STRONGLY CORRELATED PHASES}

In the previous sections, we have performed various calculations that can only work close to the Mott insulator phase. Theoretical approaches are limited for the two-dimensional Bose-Hubbard model under a magnetic field, particularly for strong fields. This is due to the complicated single-particle spectrum as well as the interplay between strong correlations and the high number of degeneracies. Both the strong-coupling and the mean-field approaches work in the region where such correlations are weak. On the other hand, this is exactly the region where novel phases are expected. For this reason, characterization of the two-dimensional Bose-Hubbard model exposed to a strong magnetic field is attracting close attention. There have been several proposals that try to connect these strongly correlated states with the formation of a vortex lattice or with the incompressible quantum liquids found in quantum Hall effects [5]. The absence of an encompassing theoretical model makes it difficult to identify the physics of this regime.

Both the strong-coupling expansion and the mean-field theory as discussed in the previous sections use the Mott insulator state as their starting point. As a result, their range of validity is limited to densities close to integer filling. In the other limit, the Gross-Pitaevskii approximation assumes a uniform gas spread over the lattice to reveal the dynamics of the system. Compared to these theoretical approaches, the DMRG has a very wide range of applicability regardless of the particle number, strength of the field, or interaction. One can calculate the ground state of the system for a finite lattice with any number of particles for all values of the magnetic field and the interaction strength. In this section, we use the DMRG method to study the two-leg Bose-Hubbard model under a magnetic field outside the Mott insulator region and look for evidence of strongly correlated behavior.

Here, we limit DMRG calculations to hard-core bosons in the infinite- $U$ limit, providing an easier implementation of the algorithm as the Hilbert space is drastically reduced by excluding multiple occupation of each site. This limit is particularly important for correlated states, as the gaps in the spectrum are expected to be more prominent with strong interactions. Within this constraint, each site is allowed to be empty or have only one boson so that the maximum occupation number $n_{\max }=1$ and the terms with the on-site interaction in the Hamiltonian become only a constraint in the Hilbert space. The Bose-Hubbard model in this limit can be mapped to a spin- $X X Z$ model, where the ground state is at half-filling. We find that our system has a ground state at half-filling not only for $\alpha=0$ but also for nonzero $\alpha$. In the two limits, all sites empty and all sites filled, the ground-state energy is 0 and the minimum of the energy is always at half-filling, which is in the middle of these two limits.

The energy gap between the ground state and the first two excited states is shown in Fig. 9 for half-filling. The figure shows that the spectrum of the three lowest lying states changes abruptly at $\alpha_{c} / \pi \approx 0.21$. This plot is symmetric around $\alpha / \pi=$ 0.5 so we only display the half. The critical value found here is consistent with the one found in the single-particle solution, which is equal to 0.2148 or 0.7852 .

To get the energies at different fillings the DMRG code must be restricted to a different particle-number-conserving subspace. We use the route proposed by Ramanan et al. [18], in which the plateaus in the chemical potential versus the density plots and the corresponding compressibility are obtained successfully. We again have a system length $L=60$ and $2 \times L=120$ sites. Beginning from $L=4$ and a total number of particles $N=4$, we increase both the lattice length and the number of particles up to where the number of particles is $N=10$. After that, the lattice length is increased while the total number of particles is held fixed at 10 . Whenever the lattice length reaches $L=60$, finite system sweeps are used to decrease the energy. Next, we increase the total particle number by 1 , keeping the system size fixed, and perform five sweeps to get the energy for this new filling. Repeating this procedure, we get energies where the particle number is increased up to $N=110$. In the end, energies of systems from $N=10$ to $N=110$ particles placed at $2 \times L=120$ sites are obtained. 

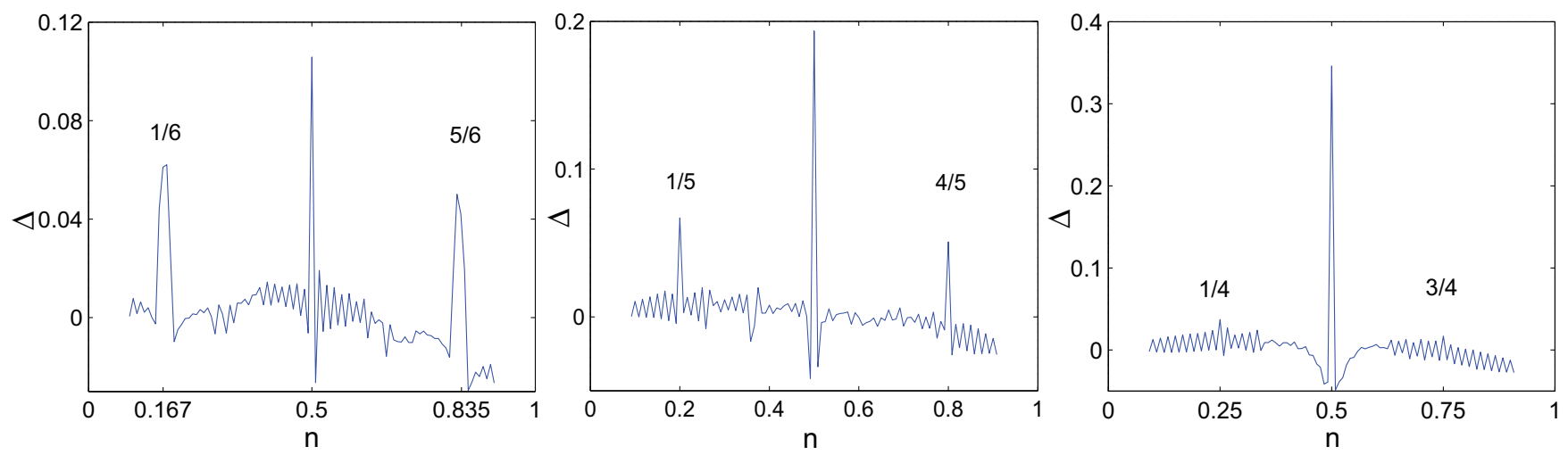

FIG. 8. (Color online) Particle-hole energy gap defined in Eq. (27) as a function of the particle density for $\alpha / \pi=1 / 3$ (left panel), $\alpha / \pi=2 / 5$ (center panel), and $\alpha / \pi=1 / 2$ (right panel). Depending on the value of $\alpha$, different peaks are seen in the energy gap that are symmetric around half-filling. Apart from the dominant peak at half-filling, $n=1 / 2$, we observe additional peaks at $n=1 / 6$ and $5 / 6$ for $\alpha / \pi=1 / 3, n=1 / 5$ and $4 / 5$ for $\alpha / \pi=2 / 5$, and $n=1 / 4$ and $3 / 4$ for $\alpha / \pi=1 / 2$.

After that, the gap formula defined by Cooper et al. [21],

$$
\Delta=N\left[\frac{E(N+1)}{N+1}+\frac{E(N-1)}{N-1}-2 \frac{E(N)}{N}\right]
$$

is used, which minimizes finite-size effects.

We show this gap for various values of magnetic field in Fig. 8. It is shown that the gap oscillates between zero and nonzero values for low densities and becomes negative towards integer filling. Apart from that there are three dominant peaks; one is always at $1 / 2$ and the other two depend on $\alpha$. The magnitudes of these changing peaks are also shown to get smaller and smaller as the field approaches $1 / 2$. It is interesting to compare these peaks by defining the filling factor [22],

$$
v=\frac{n}{f}
$$

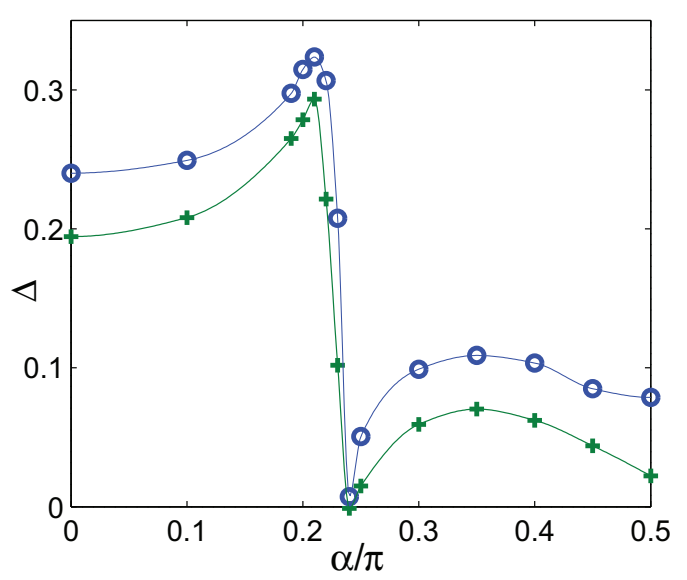

FIG. 9. (Color online) Gap between the ground state and two excited states as a function of the magnetic field at half-filling for hard-core interaction. The gap between the first excited state and the ground state $\left(E^{1}-E^{0}\right)$ is shown by (green) crosses, whereas the gap between the second excited state and the ground state $\left(E^{2}-E^{0}\right)$ is shown by (blue) circles. Thin solid lines are spline interpolations to data points. The spectrum shows a jump at $\alpha_{c} / \pi=0.21$, which is very close to the critical magnetic field calculated from the single-particle spectrum. where $n$ is the particle density and $f$ is the vortex density defined as the phase attained around a unit cell divided by $2 \pi$, which means $f=\alpha / \pi$ in our model. We see that the corresponding distinct values of the filling factors for the peaks in Fig. 8 are obtained as $v=1 / 4,3 / 4,5 / 4$ for $\alpha / \pi=1 / 3$; $v=1 / 4,5 / 8,1$ for $\alpha / \pi=2 / 5$; and $v=1 / 4,1 / 2,3 / 4$ for $\alpha / \pi=1 / 2$.

The dependence of the gap on the filling fraction is clear evidence of the role played by the interactions. However, our simple finite-size DMRG calculations cannot reveal the character of correlations within these states. Future studies of the system in this limit must include larger system sizes, finite on-site interactions, and careful consideration of finite-size effects to reveal the physics of possible correlated states in the two-leg Bose-Hubbard ladder.

\section{CONCLUSION}

Our calculations lead to a number of conclusions related to the recent experiment in Ref. [7]. The experiment probed only the limit where the number of particles per site is high, which can mostly be described by the Gross-Pitaevskii level approximations. The reported phase transition between the two phases is driven by the change in the character of the single-particle spectrum rather than interactions. In this limit, the effect of interactions is expected to be quantitative rather than qualitative. Our calculations indicate that the interactions will shift the boundary between the Meissner and the vortex phases, however, observation of this shift is complicated by the uncertainty due to the finite temperature in the experiments. A recent paper [23] argues that another effect of the interactions would be the spontaneous breaking of the symmetry between the two legs. As our calculations have this symmetry built in, we cannot investigate such a transition.

While the current experiment operates in the superfluid regime, it is natural to expect further experiments in this system to probe the region with only a few particles per site where the insulating state is likely. We expect our strong-coupling and DMRG results to be quantitatively correct for the Mott transition boundary. While the effect of the external confining potential is weak in the experiment, a wedding-cake structure 
would be a clear indication of the Mott transition. Such a wedding-cake structure not only can be observed by looking at the density but also can be deduced from the link currents investigated by the method used in the present experiment.

Finally, our DMRG results for noninteger filling factors provide some evidence for the possibility of correlated phases in this system. However, we cannot confidently assert the presence of these phases due to the finite-size limitations of our calculation. To judge the viability of the experimental observation of these phases a better characterization of their gaps and correlation properties must be made. Nonetheless, our results indicate that this regime should be interesting to investigate experimentally.

In conclusion, we have worked on the two-leg BoseHubbard ladder exposed to a magnetic field within various theoretical approaches and implemented the DMRG to study the behavior of the system. We have found that the system has two distinctively different regimes, in agreement with the recent experiment. The shape of the Mott insulator region is obtained by three methods: variational mean-field theory, strong-coupling perturbation theory, and DMRG. We found that the shape of the lobe is consistent within the DMRG and strong-coupling approximation, while the results of the meanfield theory are relatively poor. Apart from the determination of the Mott lobes, the system is found to display novel physical properties as a result of the single-particle spectrum. We believe that this model serves as an important tool for understanding the general properties of optical lattices coupled to a gauge field. In the latter part of the paper, we have calculated the excitation gap for noninteger filling and found distinct peaks at simple fractions of particle number to flux quanta. This regime will be investigated further in subsequent work.

Note added in proof. Recently, the same system was investigated theoretically in [24] and [25]. We believe that our results and these theoretical papers are complementary.

\section{ACKNOWLEDGMENTS}

A.K. thanks Levent Subaşı and Onur Umucalılar for many useful discussions and Tomotoshi Nishino and Andrej Gendiar for useful correspondence on DMRG. M.Ö.O. was supported by Türkiye Bilimsel ve Teknolojik Araştırma Kurumu (TÜBITAK) Grant No. 112T974.
[1] Y.-J. Lin, R. L. Compton, K. Jimenez-Garcia, J. V. Porto, and I. B. Spielman, Nature 462, 628 (2009).

[2] H. Miyake, G. A. Siviloglou, C. J. Kennedy, W. C. Burton, and W. Ketterle, Phys. Rev. Lett. 111, 185302 (2013).

[3] M. Aidelsburger, M. Atala, M. Lohse, J. T. Barreiro, B. Paredes, and I. Bloch, Phys. Rev. Lett. 111, 185301 (2013).

[4] D. R. Hofstadter, Phys. Rev. B 14, 2239 (1976).

[5] G. Möller and N. R. Cooper, Phys. Rev. Lett. 103, 105303 (2009); N. R. Cooper and J. Dalibard, ibid. 110, 185301 (2013).

[6] R. O. Umucalılar and M. Ö. Oktel, Phys. Rev. A 76, 055601 (2007).

[7] M. Atala, M. Aidelsburger, M. Lohse, J. T. Barreiro, B. Paredes, and I. Bloch, Nature Phys. 10, 588 (2014).

[8] A. Keles, Master's thesis, Bilkent University 2009; http://www.thesis.bilkent.edu.tr/0003932.pdf.

[9] L. D. Landau and E. M. Lifshitz, Statistical Physics: Vol. 2 (Pergamon Press, Oxford, UK, 1980).

[10] T. D. Kuhner, S. R. White, and H. Monien, Phys. Rev. B 61, 12474 (2000).

[11] T. D. Kuhner and H. Monien, Phys. Rev. B 58, R14741 (1998).
[12] M. P. A. Fisher, P. B. Weichman, G. Grinstein, and D. S. Fisher, Phys. Rev. B 40, 546 (1989).

[13] J. K. Freericks and H. Monien, Europhys. Lett. 26, 545 (1994).

[14] J. K. Freericks and H. Monien, Phys. Rev. B 53, 2691 (1996).

[15] M. Niemeyer, J. K. Freericks, and H. Monien, Phys. Rev. B 60, 2357 (1999).

[16] S. R. White, Phys. Rev. Lett. 69, 2863 (1992).

[17] S. R. White, Phys. Rev. B 48, 10345 (1993).

[18] S. Ramanan, T. Mishra, M. S. Luthra, R. V. Pai, and B. P. Das, Phys. Rev. A 79, 013625 (2009).

[19] M. S. Luthra, T. Mishra, R. V. Pai, and B. P. Das, Phys. Rev. B 78, 165104 (2008).

[20] S. R. White, Phys. Rev. B 72, 180403(R) (2005).

[21] N. R. Cooper, N. K. Wilkin, and J. M. F. Gunn, Phys. Rev. Lett. 87, 120405 (2001).

[22] N. R. Cooper, Adv. Phys. 57, 539 (2008).

[23] R. Wei and E. J. Mueller, Phys. Rev. A 89, 063617 (2014).

[24] M. Piraud, F. Heidrich-Meisner, I. P. McCulloch, S. Greschner, T. Vekua, and U. Schollwöck, arXiv:1409.7016.

[25] D. Hügel and B. Paredes, Phys. Rev. A 89, 023619 (2014). 